\title{
The Effelsberg surveys of pulse shapes
}

\author{
Jaroslaw Kijak \\ Max-Planck-Institut für Radioastronomie, Bonn, Germany \\ Astronomy Centre, Pedagogical University, Zielona Góra, Poland \\ Michael Kramer, Richard Wielebinski, Axel Jessner \\ Max-Planck-Institut für Radioastronomie, Bonn, Germany \\ John-Hugh Seiradakis \\ Department of Astrophysics, University of Thessaloniki, Greece
}

Pulse shapes of pulsars have been observed at many frequencies with the Effelsberg $100-\mathrm{m}$ radiotelescope since 1972 . The rationale behind these surveys was to use the great sensitivity of the 100-m telescope of the Max-Planck-Institut für Radioastronomie in particular at high radio frequencies for a complete study of pulse profiles.

The recent advent of HEMT amplifiers has improved the observational sensitivity enormously. Using these new systems it was possible to obtain integrated profiles of 183 pulsars at $1.4 \mathrm{GHz}, 46$ pulsars at $4.75 \mathrm{GHz}$ and 24 pulsars at 10.6 $\mathrm{GHz}$ (see Seiradakis et al. 1995). Furthermore eight pulsars have been studied at $9 \mathrm{~mm}$ wavelengths (Kramer et al. 1996).

A new sensitive HEMT system at $4.85 \mathrm{GHz}$ has recently been installed in the $100-\mathrm{m}$ radiotelescope. The system of $500 \mathrm{MHz}$ bandwidth gives $T_{\text {sys }}<30 \mathrm{~K}$ for elevations above $20^{\circ}$. We use two $8 \times 60 \mathrm{MHz}$ filter banks as a de-disperser for two circular polarizations. With this observing system we have detected more than 80 weaker pulsars at $4.85 \mathrm{GHz}$ in some $60 \mathrm{hrs}$ of observing time (Kijak et al. 1996).

We intend to go back to $1.4 \mathrm{GHz}$ and complete the pulse shapes study of all objects with $S \gtrsim 1 \mathrm{mJy}$. The $4.85 \mathrm{GHz}$ survey aims at completion at the $S \sim 0.05 \mathrm{mJy}$ level. Depending on the spectral index, additional observations at $10.5 \mathrm{GHz}$ are planned. Studies of pulsars at $32 \mathrm{GHz}$ and $43 \mathrm{GHz}$ will also be carried out with new wide-band systems at the $100-\mathrm{m}$ telescope.

\section{References}

Kijak, J. et al. 1996, in preparation

Kramer, M. et al. 1996, A\&A, 306, 867

Seiradakis, J.H. et al. 1995, A\&AS, 111, 205 
FIGURE I. The new Effelsberg survey of pulsars at $6-\mathrm{cm}$ wavelengths

PSR B0942-13

$P=0.57 \mathrm{~s}, D N=13$ pcicmi, $S=0.05 \mathrm{myy}$

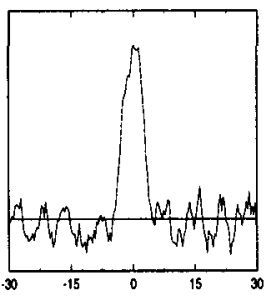

PSR B1620.09

$P=1.276 \mathrm{~s}, D M=70 \mathrm{DCC} / \mathrm{m}, \mathrm{S}=0.05 \mathrm{my}$

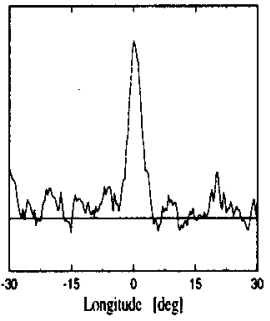

PSR B1612+07

$P=1.206 \mathrm{~s}, \quad \mathrm{NH}=21 \mathrm{gcc} / \mathrm{cm}, \mathrm{S}=0.04 \mathrm{mJy}$

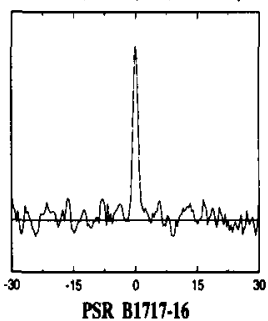

$P=1.565 \mathrm{~s}, \quad 0 \mathrm{NH}=42 \mathrm{pdcms}, \mathrm{S}=0.06 \mathrm{~mJ}$

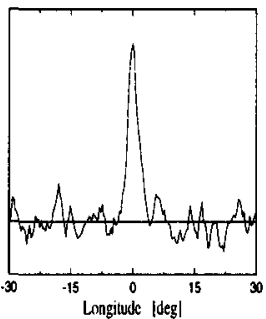

PSR B1750-24

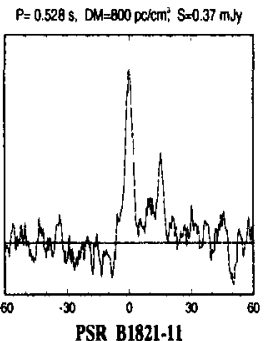

$P=0.435$ s, $\quad 0 M=620$ pcicm, $S=0.09 \mathrm{~m} / \mathrm{y}$

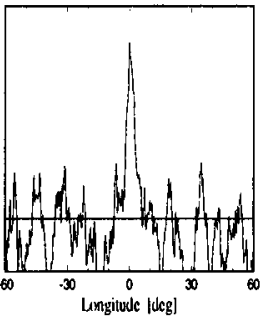

PSR B1758-23

$P=0.415 \mathrm{~s}, O M=1140 \mathrm{pccm}, S=0.43 \mathrm{mJy}$

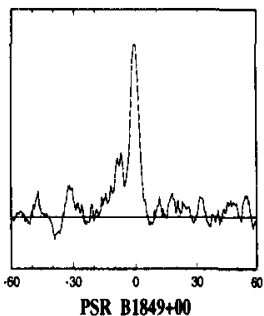

$P=2.18 \mathrm{~s}, D M=680 \mathrm{pod} / \mathrm{cm}, \mathrm{S}=0.28 \mathrm{~m} / \mathrm{y}$

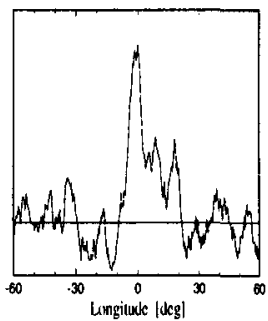

a) The weakest pulse shapes

b) Pulsars with high dispersion measure

PSR B1702-19

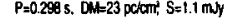

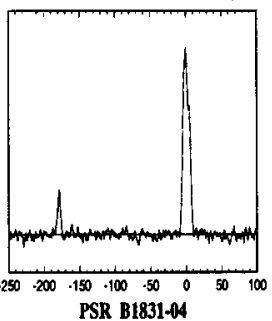

$P=0.29 \mathrm{~s}, \quad D M=79$ pecon, $S=2.11 \mathrm{~m} / \mathrm{y}$

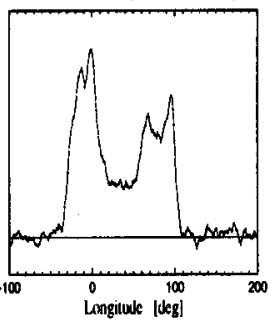

c) Some interesting pulse shapes
PSR J1022+10

$P_{2} 0.016$ s, DM=10 pcicmi: $S=0.4 \mathrm{mw}$

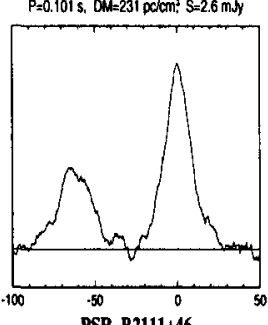

$P=1.014 \mathrm{~s}, D M=141 \mathrm{pcccm} S=1.89 \mathrm{mw}$

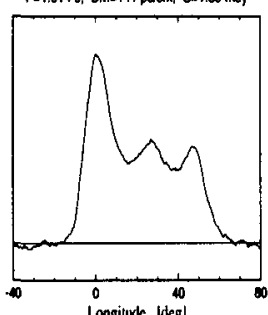

d) Millisecond pulsars
PSR J1713+07

$P=0.004 \mathrm{~s}, 0 \mathrm{~N}=16 \mathrm{pccm}, S=1.3 \mathrm{~ms}$

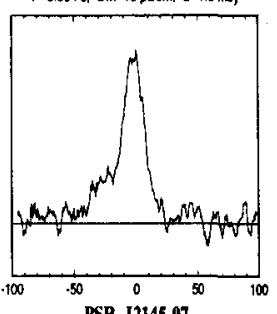

PSR J2145-07

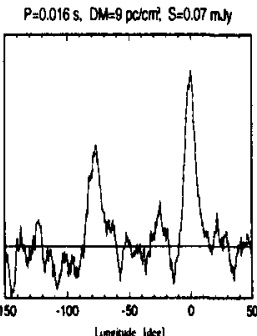

